\title{
Effects of aging on speech perception of individuals with and without Parkinson disease
}

Sabiha Parveen, Anna Slaten

Department of Communication Sciences and Disorders, Oklahoma State University, Stillwater, OK, USA

Purpose: A majority of individuals with Parkinson disease (PD) experience speech changes over time. Due to limited understanding of factors utilized by untrained listeners to identify Parkinsonian speech at discourse level, the present study examined aging effects on perception of Parkinsonian speech by three different listener groups (younger, middle-aged, and older-aged adults).

Methods: The study included five speakers with PD and five age-and-gender matched healthy control speakers. A total of 60 listeners (younger adults, middle-aged adults, and older adults) completed perceptual ratings based on six speech variables (including pitch, pitch variability, loudness, speech rate, pauses, and understandability). In addition, all listeners predicted whether or not the speaker they had listened to had PD.

Results: Older-aged listeners demonstrated significantly less than ideal ratings for loudness and understandability compared to younger and middle-aged groups. The prediction of whether or not a speaker had PD was dependent on different speech dimensions. In addition, all listener groups correctly identified the speaker with the most advanced disease severity.

Conclusions: Older-aged listeners demonstrated changes in speech perception of individuals with and without PD during quiet listening conditions. In addition, all listener groups were perceptive of voice changes secondary to PD during the perceptual rating task. Listener groups demonstrated different trends with younger adults more likely to attribute any changes in speech to a disorder while older-aged adults more likely to accept the changes in speech as normal aging effects. Overall, the study provided evidence for potential listener-assisted or listener-based rehabilitation techniques for individuals with PD.

Keywords: Perceptual speech ratings, Parkinson disease, Speakers with PD, Aging effects, Listener groups, Prediction of Parkinson disease

\section{INTRODUCTION}

\section{Listener impressions of Parkinsonian speech}

Parkinson disease (PD) is the second most common neurodegenerative disorder among the elderly [1]. It is typically associated with hypokinetic dysarthria and a majority of individuals experience some form of change in voice, articulation, and intelligibility during their disease progression [2,3]. Specific to speech intelligibility, over half of the neurologically normal listeners rated speakers with PD as "difficult to understand" and $38 \%$ of speakers with PD self-reported speech difficulties as a major concern [3]. Irrespective of disease severity, the reduced ability to communicate has detrimental effects on the family, social, and vocational life of individuals with PD $[4,5]$.

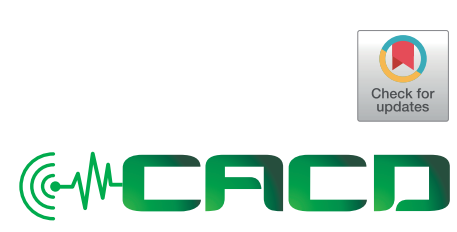

Received: May 7, 2019

Revision: August 15, 2019

Accepted: August 22, 2019

Correspondence:

Sabiha Parveen

Department of Communication Sciences and Disorders, Oklahoma State University, 042 Murray Hall, Stillwater, OK 74078 , USA

Tel: +1-405-744-5116

Fax: +1-405-744-8070

E-mail: sabiha.parveen@okstate.edu

(C) 2019 The Korean Association of SpeechLanguage Pathologists

This is an Open Access article distributed under the terms of the Creative Commons Attribution NonCommercial License (http://creativecommons.org/ licenses/by-nc/4.0/) which permits unrestricted noncommercial use, distribution, and reproduction in any medium, provided the original work is properly cited. 
With this in mind, it is important that healthcare professionals, such as speech-language pathologists (SLPs), who are evaluating and treating individuals with PD are aware of possible listening effects and other aging effects for perception of dysarthric and normal speech. In addition, creating awareness among individuals with neurological disorders as well as family members regarding listening effects can be important to achieve better rehabilitation outcomes. Therefore, the current study examines the perceptual impressions of neurologically healthy listeners for speakers with and without PD during a monologue task. The following sections summarize the existing evidence based on selected studies for listener impressions associated with Parkinsonian speech.

A large majority of existing studies have examined listener impressions based on structured speech tasks including repetition of word, phrase or sentence length materials, reading tasks, and picture description tasks. One study reported that neurologically normal listeners perceived speakers with PD to speak faster during repetition and reading tasks when compared to normal speakers [6]. In another study, negative impressions were reported among neurologically healthy listeners for speakers with PD during a picture description task with speakers termed as sounding less happy, friendly, and interested when compared to healthy speakers [7]. Specific to speech intelligibility, researchers have reported either better intelligibility among speakers with PD during reading when compared to monologues $[8,9]$ or similar performance across both reading and monologue tasks $[10,11]$. Kempler and Van Lancker observed that reading and repetition tasks were more likely to provide higher intelligibility scores due to the external cues provided to the speakers that was otherwise not available during the monologues [9]. Miller and colleagues further noted that intelligibility scores from structured reading tasks might not always accurately describe the speech problems in PD [3].

Specific to listener groups, some of the existing studies have included either untrained or trained listeners (such as practicing SLPs, graduate students, or professionals working with varying forms of dysarthria) for perception of different forms of dysarthria including ataxia [12], mixed dysarthria [9] or secondary to amyotrophic lateral sclerosis [13] and cerebral palsy [14]. In addition, some studies have examined perceptions of normal listeners of different ages for combination of dysarthric speakers $[13,15]$. However, studies examining speaker characteristics of varying severities of hypokinetic dysarthria secondary to PD by different aged listener groups (with minimal to no professional training) are rather sparse $[16,17]$.
Among the existing studies, McAuliffe and colleagues examined perception of normal listeners for phrases produced by six speakers with PD with mild-moderate hypokinetic dysarthria [16]. This study specifically examined speech intelligibility of hypokinetic speakers and indicated benefits of increased intelligibility with loud and slow conditions by normal listeners. More recently, Ma and colleagues included listener ratings for three dimensions including global severity, intelligibility and voice quality for 26 speakers with varying severities of hypokinetic dysarthria during reading and monologue tasks using a direct magnitude estimation rating task [17]. Listeners included three SLPs and the study concluded significant effects of disease severity (i.e., moderate-severe group perceived to be less intelligible than the mild hypokinetic speakers) and stimulus type (i.e., lower intelligibility of monologue compared to reading) when perceived by normal listeners. Both studies $[16,17]$ indicated the need to determine the correct balance between factors that provide detailed information about dysarthric speech perception (listeners' characteristics) as well as those that accurately reflect the day-to-day functioning of individuals with PD (as noted in monologues and conversations).

\section{Aging effects on speech perception}

Normal aging is frequently associated with deterioration and increased effort in speech identification and comprehension [18-20]. Some of the prior studies have examined effects of stimulus variability, syntactic complexity, cognitive demands, background noise, and compensatory strategies used by different listener groups when listening to neurologically healthy speakers $[18,21,22]$. Findings have been mixed with some researchers reporting similar performance of younger and older adults for sentence identification tasks in quiet conditions [23, 24] while others reporting age-related decline with poorer performance of older listeners even in the absence of hearing loss $[18,25]$. The age-related decline has been reported to be created by possible changes in auditory and cognitive processing $[18,19,22,25]$. Overall, these authors concluded that additional studies are needed to determine the practical significance of aging effects for speech processing in daily life as well as for different complexities of speech materials.

Effects related to aging have been examined among listeners of different age groups based on their perception of dysarthric speakers who in turn are often associated with marked articulatory and prosodic deficits. Several studies have examined perception of different types of dysarthric speech and 
have reported poorer performance of older listeners while listening to phrases or sentences produced by these speakers [26-29]. These studies have reported possible influence of aging on several factors including auditory discrimination, semantic knowledge, and information processing leading to overall reduced performance among older listeners for dysarthric speech [26-29]. In contrast, Poole (2011) indicated no listener group differences for perception of dysarthric speech [30]. Specifically, the author examined cognitively matched younger and older listeners and their ability to perceive sentences produced by four dysarthric speakers and one control speaker [30]. No significant differences were reported between younger and older listeners for their ratings of intelligibility (scores of transcription task), comprehensibility (answering questions regarding the stimuli), and acceptability of speakers. The author concluded that cognitive abilities of listener groups might be a pertinent factor to consider when evaluating their performance for listening tasks. In a different study, younger and older listeners were reported to perform similarly during speech perception of participants with hypokinetic dysarthria secondary to PD at phrase level [31]. These authors discussed that older listeners performed better possibly due to their larger receptive vocabularies and presumed greater language familiarity of dysarthric speech when compared to younger listeners. In conclusion, the prior evidence for aging effects among normal listeners has been mainly based on perception of phrases and sentences produced by dysarthric speakers. In addition, the existing findings for possible aging effects on neurologically normal listeners have been mixed due to differences in the task complexity, different profiles of younger and older listener groups, and selection of certain parameters which may or may not always reflect the overall speech signal of dysarthric speakers.

Most of the existing perceptual studies have used the "best" recorded speech samples during structured tasks [28]. Limited research is currently available regarding how listeners perceive normal and dysarthric speech in everyday listening contexts and environments [32]. Dagenais and colleagues recommended future studies to identify the factors of dysarthric speech that are most helpful for effective assessment and management of speech deficits [27]. The gold standard for diagnosing dysarthric speech disorders including PD is auditory perception of speech disturbances [26]. However, a majority of the existing studies have focused on selected aspects of listening experience including speech intelligibility, acceptability of the speaker and prosodic aspects such as rate of speech.
The current study thus attempts to examine listener impressions of different severities of speakers with PD during a naturalistic speech task based on six different speech dimensions including pitch, pitch variability, loudness, pauses, rate of speech, and understandability. These dimensions were selected based on prior literature suggesting changes in these areas secondary to PD $[2,3]$. In addition to the examination of perceptual ratings of $\mathrm{PD}$ speakers, the current study also aims to predict the factors that neurologically healthy listeners may utilize to make assumption regarding whether a person has PD or not. Prior studies have consistently reported changes perceived by listeners for speakers with PD but limited studies have examined what variables or factors help them distinguish PD from non-PD speakers. Specific to aging effects on listening skills, prior studies have reported progressive changes among typical younger, middle and older age groups for auditory reflexes [33], sound-source identification [34], and speech perception [20]. The existing evidence suggests that adults with typical hearing may experience changes as early as the third decade of life [34]. Thus, it is important to examine abilities of younger, middle- and older age groups for perception of Parkinsonian speech to get an overall view of possible effects on listening experiences.

\section{Aims of the study}

Due to the intricate design of the laryngeal muscles, the voice is often the first indication of a neurological disorder as well as the progression of the disorder in severity [35]. Therefore, it is important to understand the impact of voice changes among speakers with PD including their perception by other listener groups of different age ranges (younger- aged $=18$-35 years; middle- aged $=36-64$ years; older- aged $=65$ years and above) . The specific age groups were chosen based on prior studies that have examined aging changes in different listener groups for normal and dysarthric speakers $[9,11,36]$. Further rationale for the particular age range for the OA group was based on hearing screening criteria for adults aged 65 and older from American Speech-Language-Hearing Association (ASHA) [37].

The current study is important for several reasons. The rapid increase in aging population throughout the world makes it highly likely that each year there will be relatively higher number of individuals living with speech disorders of neurological origin [31]. In addition, a comprehensive understanding of how older people who are often the most common communication partners of other older individuals perform during perception of natural speech tasks has important 
consequences for both speech and audiological habilitation and rehabilitation [31]. Most of the existing studies have measured perception of dysarthric speech based on young healthy listeners. The relatively fewer studies that have included older listeners have focused on general intelligibility, acceptability, or certain aspects of the listening performance for speakers with dysarthric speech [28-30].

Therefore, the aim of the present study is to determine if younger, middle-aged, and older listeners exhibit significant differences in their ability to perceive dysarthric speech secondary to PD. The study has two research questions: (i) Are there listener group differences for younger, middle-aged and older-aged adults during perception of monologues produced by individuals with PD and age- and gender-matched normal speakers? (ii) Are there differences in the ability of different listener groups to predict whether or not a person has PD based on their speech sample? If so, what variables contribute to a listener's judgment of whether or not a speaker has PD?

Specific to the first research question, it is clearly known that hearing acuity, working memory, and vocabulary influence a person's speech perception ability. Our hypothesis is that younger and middle-aged listeners groups will perform similarly when compared to the older listeners on the perceptual speech rating task. In addition, with regard to ability to predict a speaker with PD, our hypothesis is that older listeners are more likely to predict them accurately as they have a higher likelihood of encountering dysarthric speech through personal and/or professional interactions over time compared to the other two listener groups.

\section{METHODS}

\section{Participants}

The current study included five individuals with PD (Mean= 66.4 years; $S D=10.78$, Range $=55-80$ years) and five age-andgender matched (age difference within one year) healthy controls (HC) as speakers. All speakers with PD (males=3; females $=2$ ) self-reported a diagnosis of idiopathic PD, no medical history of other neurological deficits, were on dopaminergic medication at the time of participation, were native speakers of American English, and demonstrated a score of 123 or greater on the Dementia Rating Scale-2 thereby ruling out any cognitive deficits (DRS-2) [38]. All speakers with PD were perceptually assessed based on criteria of Darley, Aronson, and Brown (1969) by an ASHA certified SLP with experience in motor speech disorders [39]. Speakers with PD demonstrated different symptoms associated with hypokinetic dysarthria including monopitch, monoloudness, harsh and breathy voice, fast speech rate, inappropriate silences, and imprecise consonants. All HC participants met the criteria of no self-reported significant medical history or neurological deficits and native speakers of American English. Table 1 includes the demographic information of all speakers. Prior to data collection, the study protocols received approval from the University Human Subjects Review Board.

Specific to listeners, the study included 20 younger-aged listeners ( males $=5$; females $=15$; mean age $=21.43$ years), 20 middle-aged listeners $($ males $=3$; females $=17$; mean age $=$ 47.29 years), and 20 older-aged listeners (males $=10$; females $=10$; mean age $=72.21$ years). All listeners self-reported

Table 1. Demographic information of speakers with Parkinson disease (PD) and neurologically normal aged- and gender-matched healthy control speakers (HC)

\begin{tabular}{|c|c|c|c|c|c|}
\hline List ID No. & Gender & Age & Severity and dysarthria type & Hearing screening & DRS-2 Score \\
\hline PD1 & Male & 67 & Moderate hypokinetic & * & 132 \\
\hline PD2 & Male & 64 & Mild hypokinetic & Passed & 144 \\
\hline PD3 & Female & 84 & Moderate hypokinetic & * & 135 \\
\hline PD4 & Female & 62 & Mild hypokinetic & Passed & 141 \\
\hline PD5 & Male & 55 & Severe hypokinetic & Passed & 128 \\
\hline HC6 & Male & 55 & $\mathrm{~N} / \mathrm{A}$ & Passed & - \\
\hline $\mathrm{HC7}$ & Female & 85 & $\mathrm{~N} / \mathrm{A}$ & Passed & 119 \\
\hline HC8 & Male & 66 & N/A & Passed & 136 \\
\hline HC9 & Female & 63 & N/A & Passed & - \\
\hline HC10 & Male & 63 & $\mathrm{~N} / \mathrm{A}$ & Passed & - \\
\hline
\end{tabular}

Columns include List of Recorded Speakers (List ID No.), Gender, Age (in Years), and Severity and Dysarthria Type.

*Hearing Screening could not be completed for these participants as they wore hearing aids during the session. Participants aged 65 and older completed the Dementia Rating Scale 2 (DRS-2). 
no medical history and functional hearing levels as determined by a hearing screening. In addition, cognitive screening was completed for all listeners except for one above 65 years and no participants were excluded due to possible cognitive deficits. Participants over 65 years were specifically screened as the prevalence of dementia doubles every 5 years after age 65 years [40]. The one participant from the OA group who was not been screened at this time lived at home independently and did not self-report any cognitive changes. The listeners self-reported different levels of experience with parkinsonian speech with 6 listeners (4 YA and 2 MA) mentioning taking prior coursework in dysarthria. These six listeners were either graduate students in speech-language pathology or SLPs primarily working with pediatric populations. In addition, a total of 24 listeners (3 YA, 8 MA, and 13 OA) self-reported knowing someone personally who was diagnosed with PD.

\section{Hearing screening}

All young aged listeners (YA) passed hearing screening for all 4 out 4 tones in both ears [37]. In addition, 13 out of 20 middle-aged listeners (MA) passed hearing screening for all 4 out 4 tones in both ears and remaining 7 listeners passed 3 out of 4 tones in at least one ear. Among the older listeners, all listeners passed 3 out of 4 tones in at least one ear. None of the listeners self-reported to be hearing aid users.

\section{Task and stimuli}

All speakers were recorded either at their respective homes or in an office space using a table top microphone (Shure SM58) attached to a digital recorder (Marantz Professional Model PMD661MKII). All speakers produced a one to two-minute monologue from a given choice of topics: job, favorite vacation, or hobby. Background noise level was measured in $\mathrm{dB}$ A with a sound level meter (BK Precision Model 732) before and during the session and was recorded to be below $40 \mathrm{~dB}$ A during all the sessions. The mouth-to-microphone distance was maintained at $15 \mathrm{~cm}$ during speech recordings for all speakers. All the recorded files were digitized at $44.1 \mathrm{kHz}$ with 16 or 32bit quantization and saved as separate WAV files. The monologues were randomized for the listening perception task.

\section{Rating task}

Listeners were presented with monologue stimuli (first 45-60 seconds of the previously recorded monologues) using a desktop computer or a laptop and a headphone set (HD280-
Professional) in a quiet laboratory space. During the listening task, the background noise of the location was measured in dB A using a Koolertron SL1361 Digital Sound Level Meter. The ambient noise level was below $40 \mathrm{~dB}$ A (mean ambient noise level = $35.56 \mathrm{~dB}$ A) during all listening sessions. Prior to the rating task, an example audio file (unrelated to the monologue stimuli) was played to make sure listeners were able to comfortably hear the presented stimuli. The volume of the computer and laptop was kept same for all sessions. All listeners heard the same sections of monologues and rated the monologues using a visual analog scale (VAS). Rating was completed using a VAS based on prior research suggesting better reproducibility and sensitivity to change for perception tasks when compared to Borg or Likert scales [41].

After listening to each monologue, each listener placed an " $\mathrm{X}$ " on a $10 \mathrm{~mm}$ line for different speech variables corresponding to personal perception of the speech/voice presented. The six speech variables included: pitch (too low/too high), pitch variability (monotone/excessive pitch variations), loudness (talking too quietly/talking too loudly), rate (too slow/too fast), pause (too short or too few/too long or too many), and speech intelligibility (not at all understandable/ completely understandable). Prior to completion of perceptual ratings, the examiner explained the rating procedure to all raters including the basic definition of each variable and its respective ideal ratings. For example, pitch was defined as highness or lowness of voice and the ideal rating for pitch was at the middle of the $10 \mathrm{~mm}$ line with two extremes of the line representing too low or too high pitch respectively. All except speech intelligibility had the ideal rating at the center of the line while the ideal rating for speech intelligibility was at the end of $10 \mathrm{~mm}$ line. Specific definitions were also provided for each variable at the bottom of the rating form for reference. In addition, the examiner provided information in case the participants asked for further clarification. No further training or examples were provided prior to the rating task as the study aimed to examine the natural abilities of different listener groups as they perceived speakers with $\mathrm{PD}$ and neurologically healthy speakers during a natural speech task. After rating each speaker on the six speech variables, all listeners answered a yes/no question related to if the speaker they just listened had a diagnosis of PD (Yes) or not (No). Table 2 includes the summary of correct positives regarding the presence or absence of PD among speakers by the different listener groups (YA, MA, and OA). 
Table 2. Descriptive data of speaker-related differences in identifying speakers with Parkinson disease (PD) by different listener groups: young adults (YA), middle-aged adults (MA) and older-aged adults (OA)

\begin{tabular}{|c|c|c|c|c|c|}
\hline Speaker group & Listener group & $\begin{array}{l}\text { Listeners who correctly } \\
\text { Identified male speakers }\end{array}$ & $\%$ Correct & $\begin{array}{l}\text { Listeners who correctly } \\
\text { identified female speakers }\end{array}$ & $\%$ Correct \\
\hline \multirow[t]{3}{*}{ Mild PD (1 M; 1 F) } & YA & $1 / 20$ & 5 & $2 / 20$ & 10 \\
\hline & MA & $1 / 20$ & 5 & $0 / 20$ & 0 \\
\hline & $\mathrm{OA}$ & $0 / 20$ & 0 & $0 / 20$ & 0 \\
\hline \multirow[t]{3}{*}{ Moderate PD (1 M; 1 F) } & YA & $8 / 20$ & 40 & $11 / 20$ & 55 \\
\hline & MA & $8 / 20$ & 40 & $9 / 20$ & 45 \\
\hline & $\mathrm{OA}$ & $7 / 20$ & 35 & $3 / 20$ & 15 \\
\hline \multirow[t]{3}{*}{ Severe PD (1 M) } & YA & $19 / 20$ & 95 & - & - \\
\hline & MA & $20 / 20$ & 100 & - & - \\
\hline & $\mathrm{OA}$ & $20 / 20$ & 100 & - & - \\
\hline
\end{tabular}

Columns include Listener Group, Total Number YES Answers Correctly Identifying Male Speaker with Mild Hypokinetic Dysarthria, Percentage of Correct YES Answers, Total Number of YES Answers Correctly Identifying Female Speaker with Mild Hypokinetic Dysarthria, and Percentage of Correct YES Answers.

\section{Reliability}

Intra-rater reliability of the listeners was completed where $10 \%$ of randomly selected listeners completed ratings for both List 1 and List 2 during the same session. No information was disclosed to these listeners at the beginning of the session regarding the fact they would rate the speakers twice during the same session. A break of approximately 5-10 minutes was taken in-between the two lists. The intraclass coefficient for the intrajudge reliability ratings was judged to be excellent at 0.917 with $95 \%$ confidence interval values between 0.895 and 0.934. In addition, the Pearson Product Moment Correlation coefficient was 0.847 for intra-rater reliability thereby indicating good level of agreement.

\section{RESULTS}

\section{Listener group differences}

A multivariate analysis of covariance (MANCOVA) was initially completed to determine listener group differences for speakers with and without PD. The six dependent variables were pitch, pitch variability, loudness, rate, pauses, and understandability. The two independent variables were listener groups (YA, MA, and OA) and speaker groups (PD and $\mathrm{HC}$ ). In addition, the MANCOVA model included familiarity with the speaker as the covariate. However, results indicated a significant factor interaction between the independent variables and the covariate. As a result, the main effects for each factor on a combined dependent variable were invalid thereby leading to violation of test assumptions for the current model [42].
As an alternative, nonparametric statistical tests were completed to examine listener and speaker group differences [43].

\section{List and order effects}

Speaker recordings were randomized into two lists (List 1 and List 2) prior to data collection. The lists were counterbalanced to minimize any order effects that might influence ratings for the speakers. Ratings from listeners from each group were sorted and compared. The alpha level was set at 0.05 for all statistical analyses. Non-parametric Kruskal-Wallis test indicated a significant difference between listeners for pitch $(p<0.001)$, pitch variation $(p<0.05)$, and rate $(p<0.005)$ based on whether they completed the List 1 or List 2 . In conclusion, there was a significant effect of list type on perceptual ratings completed by the listener groups.

\section{Listener and speaker group differences}

Independent samples Kruskal-Wallis Test indicated a significant listener group effect for loudness $(p=0.009)$, rate $(p=$ $0.004)$, pauses $(p=0.045)$, and understandability ratings $(p<$ $0.001)$. A modified alpha value of $0.008(=0.05 / 6)$ was used to interpret post-hoc group differences. Post-hoc tests (MannWhitney U test) indicated significant differences between MA and OA in terms of pauses $(p=0.001)$ and understandability ratings $(p<0.001)$. When ratings for YA and OA groups were compared, significant group differences were found for loudness $(p=0.003)$ and understandability $(p<0.001)$. However, there were no significant differences between YA and MA groups for any of the six perceptual speech variables (Figure 1). 


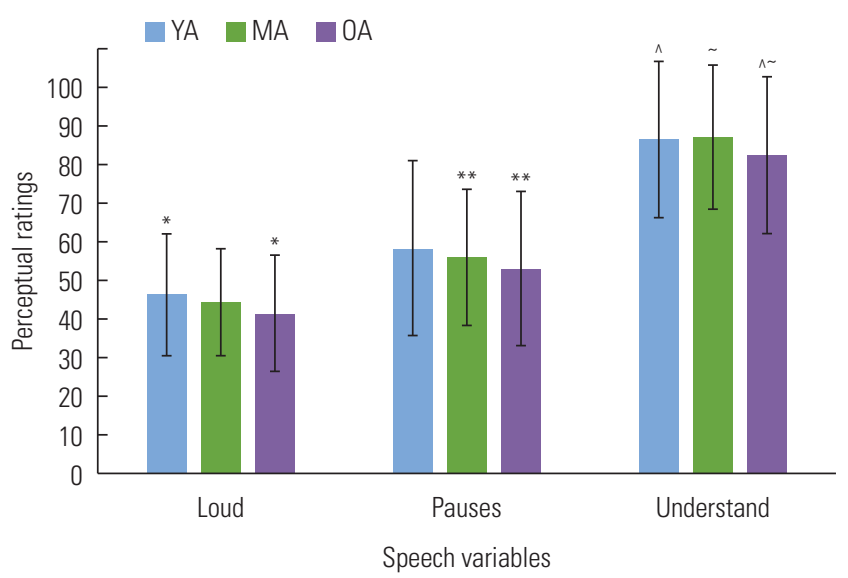

Figure 1. Listener group differences for younger aged adults (YA), Middle aged adults (MA) and older aged adults (OA). ${ }^{*} p<0.05 ;{ }^{* *} p=0.01 ;{ }^{\wedge \sim} p<0.01$.

A separate independent samples Kruskal-Wallis Test indicated significant speaker group differences for pauses $(p<0.001)$ and understandability $(p<0.001)$ ratings. Speakers with PD were associated with higher ratings for pauses (i.e., longer and frequent pauses) and lower ratings for understandability when compared to healthy speakers (Figure 2).

\section{Identification of speakers with PD}

In addition to comparison of listener groups for speakers with PD and NS, the present study examined ability of these listeners to predict whether or not the specific speaker had PD. Based on descriptive examination, the three listener groups (YA, MA, and OA) correctly identified PD speakers with $41 \%$, $38 \%$, and $30 \%$ and HC speakers with $78 \%, 86 \%$, and $93 \%$ accuracy levels respectively. All except one listener (i.e., 59/60) correctly identified the speaker with PD who was diagnosed with severe hypokinetic dysarthria. Table 2 summarizes the prediction of PD among speakers by different listener groups, showing that as impairment increased from mild to severe, the correct number of positives identifying the speaker as having PD also increased.

Due to exploratory nature of possible factors influencing a listener's response to label a person to have PD or not (a dichotomous variable), logistic regression was utilized here [43]. The dependent variable was the response to the yes/no question and the independent (or predictor) variables were listener group, speaker group, pitch ratings, loudness ratings, speech rate ratings, pause ratings, and understandability ratings. Regression results indicated that the overall model fit was questionable (-2 Log Likelihood $=531.536)$ but was statistically reliable in distinguishing presence or absence of PD in

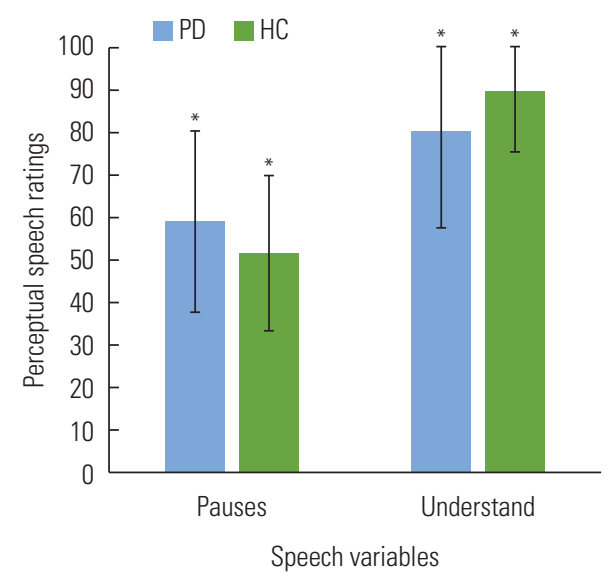

Figure 2. Group differences for individuals with Parkinson disease (PD) and control speakers $(\mathrm{HC}) .{ }^{*} p<0.001$.

a speaker; $\left[\chi^{2}(1)=143.710, p<0.001\right]$. Nagelkerke's $R^{2}$ of 0.462 indicated a moderately strong relationship between prediction and grouping. The Hosmer and Lemeshow Test was not significant thereby indicating that the model is strongly accurate in prediction of speakers with and without $\mathrm{PD}$. Based on the classification table, the model correctly classified $81.7 \%$ of participants with $\mathrm{PD}$. Odd ratios for speech rate ratings $\left(e^{B}=\right.$ $1.022)$ and understandability ratings $\left(e^{B}=1.036\right)$ revealed that increase in the likelihood of perceiving a speaker to have PD increased when the predictors increased by 1 . In conclusion, all three listener groups were more likely to predict the speakers with PD based on their perceptual ratings for speech rate and understandability.

\section{DISCUSSION}

\section{Listener group differences}

The current study investigated younger, middle-aged, and older listeners' processing of Parkinsonian speech during a monologue task. Results indicated that listeners aged 65 or above (i.e., OA group) demonstrated significantly lower than ideal ratings for loudness, pauses, and understandability when compared to younger and middle-aged listeners. The findings of older-aged listeners (who otherwise self-reported normal hearing) demonstrating changes in perception of speech stimuli in quiet listening conditions, are similar to prior studies examining age-related changes among elderly listeners [25-28]. Results from the current study provide support to the fact that aging is associated with changes in understandability of speech stimuli during quiet listening conditions $[18,25]$. A majority of prior studies have examined per- 
ception of normal listeners at word or sentence level tasks [1825]. However, the current study adds evidence to a set of limited studies $[16,17]$ that have included monologues by multiple speakers with different severities of PD and age-and-gender matched HC speakers. However, the two prior studies differed markedly in methodology from the current study. While McAuliffe et al. [16] included only two age groups (younger and older) age group, Ma et al. [17] included three expert listeners for the perceptual task. Therefore, results from the current study provides datapoints for monologues perceived across a wide range of ages (18-84 years) of majority untrained listeners.

Findings about how neurologically normal listeners perceive monologues by people with and without PD can have important treatment implications for dysarthria management. Prior studies indicate that individuals with PD have self-perception deficits specific to their speech and non-speech performance [44-46]. Treatment programs involving training of family members and health professionals for listening and identification of perceptual speech changes secondary to PD can thus be effective in early referral as well as more effective treatment outcomes. A review of literature has indicated that treatment programs focusing on neurologically healthy listeners (including communication partners, family members, and friends) thereby bypassing the dysarthric speaker and any associated conditions affecting the rehabilitation outcomes negatively, may prove key to maximizing the communication success of dysarthric speakers including those with PD [47].

The current study examined the listener impressions based on multiple speech dimensions including pitch, pitch variability, loudness, speech rate, pauses, and understandability. This is in contrast to majority of prior studies that have reported on specific aspects of performance of speakers with PD including speech intelligibility ratings [9-11], rate of speech [6], and other prosodic parameters [7]. Therefore, results from the current study provide useful information regarding the age-related speech perception changes based on various factors among different listener groups, particularly in the older-age group (i.e., 65 years and above) when there is also a higher likelihood of presbycusis and changes in speech processing for both quiet and noisy conditions.

\section{Speaker differences}

The current study indicated significant group differences among speakers with and without PD. Speakers with PD were rated to have significantly more frequent and/or longer pauses and overall less understandability compared to age-and-gender matched HC speakers. These findings are similar to a prior study where younger and older normal hearing adults rated dysarthric speakers secondary to stroke to be less intelligible during reading when compared to age-and-gender matched control speakers [27]. Both the study by Dagenais et al. [27] and the current study demonstrated that all listener groups reported high speech intelligibility ratings for normal speakers thereby approaching ceiling effects. The determination of voice changes during listening tasks can be critically important for individuals with PD. As indicated by Ho and her colleagues, voice dysfunction (including changes in voice quality and speech volume) is more frequently observed in majority of individuals with PD during early and middle stages of the disease as compared to changes in articulation and fluency [2]. Therefore, findings from the study further add to the limited existing evidence of listener impressions of early important voice markers including reduced loudness, reduced speech rate, increased pauses and reduced understandability among speakers with PD during a monologue task.

\section{Aging differences}

In addition to examining age-related changes in perception by different listener groups, the present study attempted to determine how accurate different listener groups were in determining whether or not a speaker has PD. The current study used a yes/no question to determine the listeners' ability to identify presence of PD among speakers after completion of perceptual speech ratings. As the aim of the study was exploratory in nature, logistic regression was used to identify the possible factors that impacted the listeners' responses for the prediction of speaker type. Age-related differences were seen in listener prediction of whether or not the speaker had a diagnosis of PD. Specifically, a relatively higher number of correct positive responses (i.e., correctly predict the speaker to have $\mathrm{PD}$ ) as well as false positive responses (i.e., predict the speaker to have PD when in reality they did not have PD) were found among the younger listener groups compared to the other two listener groups (i.e., middle-aged and older-aged). To the best of our knowledge, this is the first study that has examined ability of untrained listeners to identify speakers with PD based on a monologue task. Prior studies have examined other variables such as acceptability or global speech severity of speakers based on monologue tasks. However, the current study indicates that even in the absence of any ear training, listeners are able to identify speakers with more advanced 
disease severity secondary to PD.

Findings from the logistic regression model suggest that perceptual ratings for speech rate and understandability significantly helped the listeners to identify whether or not a speaker had PD. In addition, listeners of all age groups were more successful in correctly predicting the speaker with severe hypokinetic dysarthria compared to speakers with mild or moderate severities. This is again consistent with prior studies, which show that as severity of PD progresses, deficits in speech become more apparent $[6,44,45]$. It is possible that younger adults consider the speech of older adults in general to deviate from normal and have a smaller internal reference for what is normal. In contrast, older adults may have more experience with different types of speakers and are also more likely to interact with older speakers on a social level. Therefore, older adults may be more accepting of changes in speech due to normal aging rather than due to a disorder.

\section{Conclusions and future directions}

In conclusion, the present study investigated age-related differences between listener perception of monologues produced by individuals with PD and age-and-gender matched neurologically normal speakers. Listener groups of different age ranges performed similarly for the perception of monologues produced by speakers of different severities of PD as well as HC. However, speakers with PD were rated to be less loud, with relatively greater number of pauses, and less understandable by all listener groups when compared to the HC group. In addition, age-related trends were observed in the prediction of whether or not the speaker had PD, such that younger listeners were more likely to have false positive responses for speakers with PD when compared to older-aged listeners. These findings suggest that younger and older-aged adults have certain perceptual expectations for typical speech changes in older adults versus changes due to a progressive and neurological disorder such as PD.

It is important to acknowledge some of the limitations of the current study. The present study recruited only five speakers with PD and five neurologically normal age- and gendermatched speakers and 60 listeners divided into three age groups (YA, MA, and OA). While the present study had a similar or larger sample size than some of the earlier listener perception studies $(26,28,30)$, and used different severities of PD, a larger sample size of speakers with different severities of PD would perhaps have more clearly shown age-related differences between listener groups. A second limitation could be the different levels of experience among listener groups for parkinsonian speech. Approximately $40 \%$ of listeners reported at least some familiarity with someone with PD which may have influenced some of their responses for the perceptual listening task. It was extremely difficult to locate listeners in the middle-aged or older-aged groups to have minimal or no prior experience with Parkinsonian speech based on the prevalence of $\mathrm{PD}$ among people aged 65 or above. It is more likely that older listeners would have some personal and/or professional interactions with individuals with PD who are also more likely to be in that similar age group (of 65 years or older). A third limitation could be the information included in the speaker monologues. Although all listeners were blinded to the speakers' identity and presence or absence of a diagnosis of $\mathrm{PD}$, it is possible that speakers may have provided personal information in the monologues that might have influenced the listener ratings and the prediction of whether the person had PD or not. A final limitation of the study was the effect of presentation list (List 1 vs. List 2) where the order of speakers may have influenced the listeners' ratings and therefore made the findings less generalizable.

Findings from the present study suggest multiple future directions. The current study did not specifically examine the role of training and/or familiarity among neurologically healthy listeners for parkinsonian speech. Future studies may consider grouping different aged listener groups with different levels of familiarity to see if longer periods of familiarity are associated with easier listening experiences for these listener groups. There are currently limited studies regarding age-related differences in speech perception on features such as pitch, pitch variability, loudness, speech rate and pauses. Future studies may consider examining age-related differences of these perceptual features using realistic speech stimuli (monologues and conversation) in conditions of both quiet and everyday noise in order to establish if aging-related differences exist for these measures in different listening conditions. Additionally, future studies may also consider recruiting a larger sample size of speakers with PD with different disease severities in order to increase the power of the study and better examine perceptual abilities by different listener groups.

\section{CONFLICT OF INTEREST}

The authors report no conflicts of interest. Both authors are responsible for the content and writing of the paper. 


\section{ACKNOWLEDGEMENT}

None to disclose.

\section{REFERENCES}

1. De Lau LM, Breteler MM. Epidemiology of Parkinson's disease. Lancet Neurology. 2006;5:525-535.

2. Ho AK, Iansek R, Marigliani C, Bradshaw JL, Gates S. Speech impairment in a large sample of patients with Parkinson's disease. Behavorial Neurology. 1998;11:131-137.

3. Miller N, Allcock L, Jones D, Noble E, Hildreth AJ, Burn DJ. Prevalence and pattern of perceived intelligibility changes in Parkinson's disease. Journal of Neurology \& Neurosurgery. 2007;78:1188-1190.

4. Majdinasab F, Karkheiran S, Moradi N, Shahid GA, Salehi M. Relation between Voice Handicap Index (VHI) and disease severity in Iranian patients with Parkinson disease. Medical Journal of Islam Republic of Iran. 2012;26:157-163.

5. Schrag A, Jahanshahi M, Quinn N. How does Parkinson's disease affect quality of life? A comparison with quality of life in the general population. Movement Disorders. 2000;15:1112-1118.

6. Tjaden K. A preliminary study of factors influencing perception of articulatory rate in Parkinson disease. Journal of Speech Language \& Hearing Research. 2000;43:997-1010.

7. Jaywant A, Pell MD. Listener impressions of speakers with Parkinson's disease. Journal of International Neuropsychological Society. 2010;16:49-57.

8. Frearson B. A comparison of the AIDS sentence list and spontaneous speech intelligibility scores for dysarthric speech. Australian Journal of Human Communication Disorders. 1985:13:5-21.

9. Kempler D, Van Lancker D. Effect of speech task on intelligibility in dysarthria: A case study of Parkinson's disease. Brain \& Language. 2002;80:449-464.

10. Bunton K, Keintz CK. The use of dual-task paradigm for assessing speech intelligibility in clients with Parkinson disease. Journal of Medical Speech Language Pathology. 2008;16:141-155.

11. Tjaden K, Wilding G. Effects of speaking task on intelligibility in Parkinson's disease. Clinical Linguistics \& Phonetics. 2011;25:155168.

12. Spencer KA, France AA. Perceptual ratings of subgroups of ataxic dysarthria. International Journal of Language \& Communication Disorders. 2016;51:430-441.

13. Dagenais PA, Brown GR, Moore RE. Speech rate effects upon intelligibility and acceptability of dysarthric speech. Clinical Linguistics \& Phonetics. 2006;20:141-148.

14. Hustad KC. Effects of speech stimuli and dysarthria severity on intelligibility scores and listener confidence ratings for speakers with cerebral palsy. Folia Phoniatrica Logopedica. 2007;59:306-317.

15. McAuliffe MJ, Fletcher AR, Kerr SE, O'Beirne GA, Anderson T. Effect of dysarthria type, speaking condition, and listener age on speech intelligibility. American Journal of Speech Language Pathology. 2016;1-11.
16. McAuliffe MJ, Gibson EMR, Kerr SE, Anderson T, LaShell PJ. Vocabulary influences older and younger listeners' processing of dysarthric speech. Journal of Acoustical Society of America. 2013;134: 1358-1368.

17. Ma JKY, Schneider CB, Hoffman R, Storch A. Speech prosody across stimulus types for individuals with Parkinson's disease. Journal of Parkinson's Disease. 2015;5:291-299.

18. Fullgrabe C, Moore BCJ, Stone MA. Age-group differences in speech identification despite matched audiometrically normal hearing: Contributions from auditory temporal processing and cognition. Frontiers Aging in Neurosciences. 2015;6:1-25.

19. Roberts KL, Allen HA. Perception and cognition in the ageing brain: A brief review of the short- and long-term links between perceptual and cognitive decline. Frontiers Aging in Neurosciences. 2016;8:39.

20. Pichora-Fuller MK, Souza PE. Effects of aging on auditory processing of speech. International Journal of Audiology. 2003;42:2S11S216.

21. Meister H, Schreitmuller S, Grugel L. Cognitive resources related to speech recognition with a competing talker in young and older listeners. Neuroscience. 2013;232:74-82.

22. Rajan R, Cainer KE. Ageing without hearing loss or cognitive impairment causes a decrease in speech intelligibility only in informational maskers. Neuroscience. 2008;154:784-795.

23. Gelfand SA, Piper N, Silman S. Consonant recognition in quiet and in noise with aging among normal hearing listeners. Journal of Acoustical Society of America. 1986;80:1589-1598.

24. Helfer KS, Freyman RL. Stimulus and listener factors affecting age-related changes in competing speech perception. Journal of Acoustical Society of America. 2014;136:748-759.

25. Cervera TC, Soler MJ, Dasi C, Ruiz JC. Speech recognition and working memory capacity in young-elderly listeners: Effects of hearing sensitivity. Canadian Journal of Experimental Psychology. 2009;63:216.

26. Garcia JM, Hayden M. Young and older listener understanding of a person with severe dysarthria. Journal of Medical Speech Language Pathology. 1999;7:109-112.

27. Dagenais PA, Watts CR, Turnage LM, Kennedy S. Intelligibility and acceptability of moderately dysarthric speech by three types of listeners. Journal of Medical Speech Language Pathology. 1999:7:9196.

28. Dagenais PA, Adlington LM, Evans KJ. Intelligibility, comprehensibility, and acceptability of dysarthric speech by older and younger listeners. Journal of Medical Speech Language Pathology. 2011;19: 37-48.

29. McAuliffe MJ, Fletcher AR, Kerr SE, O'Beirne GA, Anderson T. Effect of dysarthria type, speaking condition, and listener age on speech intelligibility. American Journal of Speech Language Pathology. 2016;26:1-11.

30. Poole RD. A comparison of intelligibility, comprehensibility and acceptability of typical dysarthric speech by younger and older normal adults (Masters' thesis). Available from ProQuest Dissertations and Theses database. 2011;(UMI 1496745). 
31. McAuliffe MJ, Gibson EMR, Kerr SE, Anderson T, LaShell PJ. Vocabulary influences older and younger listeners' processing of dysarthric speech. Journal of Acoustical Society of America. 2013; 134:1358-1368.

32. McAuliffe MJ, Borrie SA, Good PV, Hughes LE. Consideration of the listener in the assessment and treatment of dysarthria. ACQuiring knowledge in Speech, Language and Hearing. 2010;12:1619.

33. Abdala C, Dhar S, Ahmadi M, Luo P. Aging of the medial olivocochlear reflex and associations with speech perception. Journal of Acoustical Society of America. 2014;2:754-765.

34. Abel SM, Giguere C, Consoli A, Papsin BC. The effect of aging on horizontal plane sound localization. Journal of Acoustical Society of America. 2000;2:743-752.

35. Duffy JR. Motor speech disorders: Substrates, differential diagnosis, and management. St. Louis, MO: Elsevier Mosby, 2005.

36. Fogerty D, Kewley-Port D, Humes LE. Temporal offset judgments for concurrent vowel by young, middle-aged, and older adults. Journal of Acoustical Society of America. 2012;6:499-505.

37. Roeser RJ, Clark JL. Pure Tone Tests. In R.J. Roeser, M. Valente, \& H. Hosford-Dunn (Eds.), Audiology: Diagnosis Volume 1. 2nd Edition (pp. 259). New York, NY: Thieme Medical Publishers. 2007.

38. Mattis S. Dementia rating scale 2 (DRS-2), 2004: Lutz, FL: PAR.

39. Darley FL, Aronson AE, Brown JR. Differential diagnostic patterns of dysarthria. Journal of Speech, Language \& Hearing Research. 1969;12:246-269.
40. Jorn AF, Korten AE, Henderson AS. The prevalence of dementia: A quantitative integration of the literature. Acta Psychiatrica Scandivanica. 1987;76:465-479.

41. Grant S, Aitchison T, Henderson E, Christie J, Zare S, McMurray J, et al. Comparison of the reproducibility and the sensitivity to change of visual analogue scales, borg scales, and likert scales in normal subjects during submaximal exercise. Chest. 1999;116: 1208-1217.

42. Mertler CA, Vannatta RA. Advanced and multivariate statistical methods. Pyrczak Publishing. Fourth Edition. 2010.

43. Adams KA, Lawrence EK. Research methods, statistics, and applications. SAGE Publications. Second Edition. 2018.

44. Clark JP, Adams SG, Dyskstra AD, Moodie S, Jog M. Loudness perception and speech intensity control in Parkinson's disease. Journal of Communication Disorders. 2014;51:1-12.

45. Parveen S. Comparison of self and proxy ratings for motor performance of individuals with Parkinson disease. Brain \& Cognition. 2016;103:62-69.

46. Parveen S, Goberman AM. Perception of speech and non-speech motor performance by individuals with $\mathrm{PD}$, communication partners, and a trained rater. Seventeenth Biennial Conference on Motor Speech: Motor Speech Disorders \& Speech Motor Control, Sarasota, FL. 2014.

47. Borrie SA, McAuliffe MJ, Liss JM. Perceptual learning of dysarthric speech: A review of experimental studies. Journal of Speech, Language, and Hearing Research. 2012;55:290-305. 